Pacific Journal of Mathematics

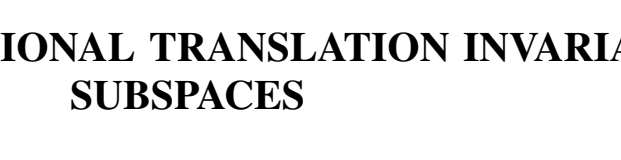




\section{FINITE DIMENSIONAL TRANSLATION INVARIANT SUBSPACES}

\section{MARTIN ENGERT}

It is known that every finite dimensional translation invariant subspace of the continuous functions on the real line consists of exponential polynomials. The purpose of this paper is to prove an analogous result under the hypotheses that the functions involved are measurable instead of continuous (and two functions are considered identical if they are equal almost everywhere) and that the functions are defined on a $\sigma$-compact locally compact abelian group. There is an application of this theorem to the characterization of differential operators at the end of the paper.

Proofs of the result for continuous functions can be found in Anselone and Korevaar [1] and Loewner [5] along with generalizations to the case of distributions in [1] and some applications in [5]. Also, a theorem of this type has been proved by Stone [6] for continuous functions on semi-groups.

Preliminary Definitions. If $G$ is a locally compact abelian group then an additive or a linear function on $G$ is a complex valued function $b$ such that $b(x+y)=b(x)+b(y)$ for all $x$ and $y$ in $G$. A polynomial on $G$ is a function of the form $p(x)=P\left(b_{1}(x), \cdots, b_{n}(x)\right)$ where $P$ is a polynomial in $n$ variables and $b_{i}(i=1, \cdots, n)$ is a continuous additive function on $G$. An exponential on $G$ is a continuous multiplicative complex valued function on $G$. An exponential polynomial on $G$ is a function of the form $\sum_{1}^{n} p_{i} g_{i}$ where $p_{i}$ is a polynomial and $g_{i}$ is an exponential.

In this paper, $G$ will always refer to a $\sigma$-compact locally compact abelian group with Haar measure $\mu$. Two measurable functions will be considered to be equal if they are equal almost everywhere.

A multi-index $p=\left(p_{1}, \cdots, p_{n}\right)$ is an $n$-tuple of nonnegative integers, and if $b=\left(b_{1}, \cdots, b_{n}\right)$ is a complex $n$-tuple, then $b^{p}$ is defined to be $b_{1}^{p_{1}} b_{2}^{p_{2}} \cdots b_{n}^{p_{n}}$. (For more details on the notation, see Hörmander [2], particularly pages $9-10$ ).

The symbol $T_{X}$ denotes the operator of translation by $x$, that is, $\left(T_{X} f\right)(y)=f(x+y)$.

LeMma 1. Let $b$ be a measurable function on $G$ and suppose that for almost every $x$ in $G$

$$
b(x)+b(y)-b(x+y)=0 \text { a.e. in } y .
$$


Then $b$ is equal almost everywhere to a continuous linear function $b^{\prime}$.

Proof. We can assume that $b$ is real, for if not we can apply the lemma to $\operatorname{Re}(b)$ and $\operatorname{Im}(b)$ separately. We shall first show that $b$ is essentially bounded on every compact subset of $G$. It is enough to show that $b$ is essentially bounded on every symmetric neighborhood of the identity with compact closure. Let $A$ be such a neighborhood and let $B=A+A$. Suppose $b$ is not bounded on $A$. Then the measure of the set $A \cap\{x: b(x)>n\}$ is positive for each positive integer $n$. Let $\left\{x_{n}\right\}$ be a sequence of points in the set $A \cap\{x: b(x)+$ $b(y)-b(x+y)=0$ a.e. in $y$ such that $b\left(x_{n}\right)>n$. We have for any $a$ in $A$ and any positive integer $n$

$$
\{x: b(x+a)>n \text { and } x \in A\} \subset\{x: b(x)>n \text { and } x \in A+A\} .
$$

So

$$
\mu\{x: b(x+a)>n \text { and } x \in A\} \leqq \mu\{x: b(x)>n \text { and } x \in B\} .
$$

Since $b$ is measurable and $B$ has finite measure, the right side goes to zero as $n$ increases, so the left side must also, that is, for any $\varepsilon>0$ there exists an $n$ so that for any $a$ in $A, \mu\{x: b(x+a)>n$ and $x \in A\}<\varepsilon$. But by choosing $a=x_{n}$ we know that $b\left(x_{n}\right)+b(x)=b\left(x+x_{n}\right)$ a.e. in $x$, so $\mu\left\{x: b(x)>n-b\left(x_{n}\right)\right.$ and $\left.x \in A\right\} \geqq \mu\{x: b(x)>0$ and $x \in A\}$. The right side of this inequality does not go to zero, so we have a contradiction.

To get a continuous function, we let $f(x)$ be a nonnegative continuous function with compact support. Then $f * b$ is continuous and

$$
(f * b)(z)=\int b(z-t) f(t) d t=b(z) \int f(t) d t+\int b(-t) f(t) d t
$$

a.e. in $z$. So

$$
b(z)=\left((f * b)(z)-\int b(-t) f(t) d t\right) /\left(\int f(t) d t\right)
$$

a.e. in $z$. If we call the right side of this equation $b^{\prime}(z)$ we have $b(z)=b^{\prime}(z)$ a.e. and $b^{\prime}(z)$ is continuous. Thus $b^{\prime}(x)+b^{\prime}(y)-b^{\prime}(x+y)$ is continuous on $G \times G$ and is equal to zero a.e. with respect to the product measure. This implies that $b^{\prime}(x)+b^{\prime}(y)-b^{\prime}(x+y)$ equals zero everywhere or that $b^{\prime}$ is linear.

LEMMA 2. Let $f$ be a measurable function on $G$. Suppose $T_{X} f-f=0$ a.e. for each $x$ in $G$. Then there is a constant $K$ such that $f(x)=K$ a.e.

Proof. Define $g$ on $G \times G$ by $g(x, y)=f(x+y)-f(x)$. Then for 
each $y, g(x, y)=0$ a.e. so $g=0$ a.e. on $G \times G$. Define $g^{\prime}(x, y)=g(x, y-x)$. (This is also equal to $f(y)-f(x))$. Since $(x, y) \rightarrow(x, y-x)$ is a one to one measure preserving map of $G \times G$ into itself, $g^{\prime}$ is also zero almost everywhere on $G \times G$. So, by Fubini's theorem, for almost all $y, g^{\prime}(x, y)=0$ a.e. in $x$. Let $y_{0}$ be such a value of $y$. Then $g^{\prime}\left(x, y_{0}\right)=$ $f\left(y_{0}\right)-f(x)=0$ a.e. Let $f\left(y_{0}\right)=K$.

Lemma 3. Suppose $f$ is a measurable function on $G$ and $T_{X} f$ $f=b(x) 1$ a.e., where $b(x) 1$ denotes the function that is the constant $b(x)$. Then $b$ is a continuous linear function on $G$.

Proof. We first show that $b$ is linear. We have $T_{X+y} f=T_{X}\left(T_{y} f\right)=$ $T_{x}(f+b(y) 1)=f+b(x) 1+b(y) 1$. But $T_{x+y} f$ is also equal to $f+$ $b(x+y) 1$. Thus $b$ is linear.

Let $g$ be an integrable function such that the integral of $g$ is 1 and $f g$ is integrable. Let $f_{n}$ be the function defined by

$$
f_{n}(x)=\left\{\begin{array}{lll}
f(x) & \text { if } & |f(x)| \leqq n \\
0 & \text { if } & |f(x)|>n
\end{array}\right.
$$

Then

$$
\int g(y)(f(x+y)-f(y)) d y=b(x)
$$

Define $b_{n}(x)$ as

$$
\int g(y)\left(f_{n}(x+y)-f(y)\right) d y \text {. }
$$

Since $f_{n}$ is bounded and $g$ is integrable, $b_{n}$ is continuous. By the Lebesgue dominated convergence theorem, $b_{n}$ converges to $b$, so $b$ is the limit of continuous functions and thus measurable.

By Lemma $1, b$ is equal almost everywhere to a continuous linear function $b^{\prime}$. Then for each $x$ in $G, b(x+y)=b^{\prime}(x+y)$ a.e. in $y$ which in turn implies that $b(x)=b^{\prime}(x)$. Thus $b=b^{\prime}$ everywhere or $b$ is a continuous linear function.

LEMmA 4. If $b=\left(b_{1}, \cdots, b_{n}\right)$ is an $n$-tuple of linear functions on $G$ and $p$ is a multi-index, then

$$
T_{x} b^{p}=p ! \sum_{q \leqq p} \frac{b^{p-q}(x) b^{q}}{(p-q) ! q !}=b^{p}+p ! \sum_{q<p} \frac{b^{p-q}(x) b^{q}}{(p-q) ! q !} .
$$

Proof. The proof of this is straightforward computation of $b^{p}(x+y)$ using the binomial theorem repeatedly, so it will be omitted here. 
LEMMA 5. Let $f$ be a measurable function on $G$ such that the complex linear space spanned by $\left\{T_{x} f-f: x \in G\right\}$ is a finite dimensional space $V$ of polynomials. Then $f$ is equal almost everywhere to a polynomial on $G$.

Proof. It might be helpful to outline the proof first. We will first construct a polynomial $f^{\prime}$ so that $\left\{T_{x}\left(f-f^{\prime}\right)-\left(f-f^{\prime}\right): x \in G\right\}$ spans a finite dimensional space of polynomials of degree less than or equal to one. We then show that if $f^{\prime \prime}$ is a function such that $\left\{T_{x} f^{\prime \prime}-f^{\prime \prime}: x \in G\right\}$ spans a finite dimensional space of polynomials of degree less than or equal to one then we can construct a polynomial $f^{\prime \prime \prime}$ so that for each $x, T_{x}\left(f^{\prime \prime}-f^{\prime \prime \prime}\right)-\left(f^{\prime \prime}-f^{\prime \prime \prime}\right)$ is equal almost everywhere to a constant. We then use the previous lemmas to conclude that $f^{\prime \prime}-f^{\prime \prime \prime}$ is equal almost everywhere to a polynomial.

First choose a finite set of continuous linear functions so that all polynomials in $V$ are built up from these linear functions. We can then choose a set $\left\{b_{1}, \cdots, b_{n}\right\}$ of linear combinations of these functions and a set of points $\left\{y_{1}, \cdots, y_{n}\right\}$ in $G$ so that $b_{i}\left(y_{i}\right)=\delta_{i j}$ and $V$ is contained in the linear space spanned by $b^{p}$ for $p$ contained in a finite set of multi-indices:.

There are thus functions $c_{p}$, defined everywhere on $G$, so that $T_{x} f-f=\sum c_{p}(x) b^{p}$. We compute

$$
\begin{aligned}
T_{x+x} f & =T_{x} T_{y} f=T_{x}\left(f+\sum c_{p}(y) b^{p}\right) \\
& =f+\sum c_{p}(x) b^{p}+\sum c_{p}(y) \sum_{r \leq p} p ! \frac{b^{p-r}(x) b^{r}}{(p-r) ! r !}
\end{aligned}
$$

with these equations holding almost everywhere on $G$. Since the functions $b^{r}$ are linearly independent, the coefficient of $b^{r}$ is equal to

$$
\begin{aligned}
& \sum_{p \geqq r} c_{p}(y) \frac{p !}{(p-r) ! r !} b^{p-r}(x)+c_{r}(x) \\
= & \sum_{p>r} c_{p}(y) \frac{p !}{(p-r) ! r !} b^{p-r}(x)+c_{r}(y)+c_{r}(x) .
\end{aligned}
$$

This expression has to be symmetric in $x$ and $y$, giving for each $r$ the set of equations

$$
\sum_{p>r} c_{p}(y) \frac{p !}{(p-r) ! r !} b^{p-r}(x)=\sum_{p>r} c_{p}(x) \frac{p !}{(p-r) ! r !} b^{p-r}(y) .
$$

Now change notation, on the right hand side let $q=p-r$. We get

$$
\sum_{p>r} c_{p}(y) \frac{p !}{(p-r) ! r !} b^{p-r}(x)=\sum_{q>0} c_{q+r}(x) \frac{(q+r) !}{q ! r !} b^{q}(y) .
$$


The sum on the right is a finite sum, say over $q=q_{1}, \cdots, q_{m}$. Choose $x_{1}, \cdots, x_{m}$ so that the matrix $\left[b_{i j}\right]=\left[b^{q_{i}}\left(x_{j}\right)\right]$ is invertible. (This is possible because the monomials $b^{q}$ are linearly independent). This is equivalent to saying that there exist numbers $B_{q, j}$ with the property

$$
\sum_{j} b^{p}\left(x_{j}\right) B_{q, j}=\left\{\begin{array}{ll}
1 & \text { if } p=q \\
0 & \text { if } p \neq q
\end{array} .\right.
$$

By letting $y=x_{1}, \cdots, x_{m}$ successively in equation (1) above we get for each $r$ a set of $m$ equations which we can solve for $[(q+r) ! / q ! r !] c_{q+r}(x)$, finding it equal to

$$
\sum_{k} B_{q, k} \sum_{p>r} c_{p}\left(x_{k}\right) \frac{q !}{(q-r) ! r !} b^{p-r}(x) .
$$

Thus, if $q>0$

$$
\begin{aligned}
c_{q+r}(x) & =\sum_{p>r} \frac{q !}{(q+r) !} \sum_{k} B_{q, k} c_{p}\left(x_{k}\right) \frac{q !}{(p-r) !} b^{p-r}(x) \\
& =\sum_{p>r}\left[\sum_{k} B_{q, k} c_{p}\left(x_{k}\right) \frac{p ! q !}{(p+q) !}\right] \frac{(p+q) ! b^{p-r}(x)}{(q+r) !(p-r) !} .
\end{aligned}
$$

Define $k(p, q)$ to be the quantity within the brackets. Note that $k(p, q)$ is defined only if $p>0$ and $q>0$. If we take the special case $r=0$ we get, for $q>0$

$$
c_{q}(x)=\sum_{p>0}[k(p, q)] \frac{(p+q) !}{p ! q !} b^{p}(x)
$$

which says that $c_{q}$ is a linear combination of monomials $b^{p}$ and that $k(p, q)$ is the coefficient of the monomial $[(p+q) ! / p ! q !] b^{p}$ in the function $c_{q}$.

We want to show that $k(p, q)$ depends only on $p+q$. We shall first show this in the case where $|p+q| \geqq 3$. Then we shall consider the case where $|p+q|=2$. Since both $p$ and $q$ in the above equation are always $>0$, the case $|p+q|=1$ does not arise.

Suppose we have $q=q^{\prime}+r$ and $p=p^{\prime}-r$. Then we want to show that $k(p, q)=k\left(p^{\prime}, q^{\prime}\right)$. But using equation (2) twice

$$
\begin{aligned}
c_{q}(x) & =\sum_{p>0}[k(p, q)] \frac{(p+q) !}{p ! q !} b^{p}(x) \\
& =c_{q^{\prime}+r}(x) \\
& =\sum_{p^{\prime}>r}\left[k\left(p^{\prime}, q^{\prime}\right)\right] \frac{\left(p^{\prime}+q^{\prime}\right) !}{\left(q^{\prime}+r\right) !\left(p^{\prime}-r\right) !} b^{p^{\prime}-r}(x) \\
& =\sum_{p^{\prime}>r}\left[k\left(p^{\prime}, q^{\prime}\right)\right] \frac{(p+q) !}{p ! q !} b^{p}(x) .
\end{aligned}
$$


So $k(p, q)=k\left(p^{\prime}, q^{\prime}\right)$ since they both can be described as the coefficient of $[(p+q) ! / p ! q !] b^{p}$ in $c_{q}$.

From this we can conclude that $k\left(p_{1}, q_{1}\right)=k\left(p_{2}, q_{2}\right)$ whenever we can find a third pair of nonzero multiindices $\left(p_{3}, q_{3}\right)$ so that $p_{1}+q_{1}=$ $p_{2}+q_{2}=p_{3}+q_{3}$ and we can compare $q_{3}$ to both $q_{1}$ and $q_{2}$ in the way we have just done. By simple arguments it can be shown that if the multi-index $s$ has $|s| \geqq 3$ and $p_{1}+q_{1}=p_{2}+q_{2}=s$ and if none of the multi-indices $p_{1}, p_{2}, q_{1}, q_{2}$ is equal to zero, then $k\left(p_{1}, q_{1}\right)=k\left(p_{2}, q_{2}\right)$.

Thus, for $|s| \geqq 3$ we can define $k(s)=k(p, q)$ for any $p$ and $q$ with $p+q=s$.

Let $f^{\prime}=\sum_{s \geq 3} k(s) b^{s}$. Then

$$
T_{x} f^{\prime}-f^{\prime}=\sum_{s \geq 3} k(s) \sum_{q<s} \frac{s !}{(s-q) ! q !} b^{s-q}(x) b^{q} .
$$

By comparing this equation (3) we can see that there are numbers $k^{\prime}(p, q)$ so that

$$
T_{x}\left(f-f^{\prime}\right)-\left(f-f^{\prime}\right)=\sum k^{\prime}(p, q) \frac{(p+q) !}{p ! q !} b^{p}(x) b^{q}+c_{0}(x) 1,
$$

where the sum is taken over the multi-indices $p$ and $q$ with $|p+q| \leqq 2$ and $p>0$. The right side of this equation contains only linear functions and constants, that is, we can define functions $d_{0}, d_{1}, \cdots, d_{n}$ so that

$$
T_{x}\left(f-f^{\prime}\right)-\left(f-f^{\prime}\right)=d_{0}(x) 1+\sum d_{k}(x) b_{k} .
$$

Let $f^{\prime \prime}=f-f^{\prime}$ for short.

Using the same trick as before:

$$
\begin{aligned}
T_{x} T_{y} f^{\prime \prime}= & T_{x}\left(f^{\prime \prime}+d_{0}(y) 1+\sum d_{k}(y) b_{k}\right) \\
= & f^{\prime \prime}+d_{0}(x) 1+d_{0}(y) 1+\sum d_{k}(y) b_{k} \\
& +\sum d_{k}(x) b_{k}+\sum b_{k}(x) d_{k}(y) 1 .
\end{aligned}
$$

This has to be symmetric in $x$ and $y$, so we get

$$
\sum d_{k}(x) b_{k}(y)=\sum d_{k}(y) b_{k}(x) \text {. }
$$

Pich $y=y_{1}, \cdots, y_{n}$ in succession, where these are the numbers chosen at the beginning of the proof so that $b_{k}\left(y_{i}\right)=\delta_{k i}$. We get

$$
d_{k}(x)=\sum_{j} d_{j}\left(y_{k}\right) b_{j}(x)
$$

and thus, by letting $x=y_{i}, d_{k}\left(y_{i}\right)=d_{i}\left(y_{k}\right)$. Let $f^{\prime \prime \prime}=(1 / 2) \sum_{i, k} d_{k}\left(y_{i}\right) b_{i} b_{k}$. Then 


$$
\begin{aligned}
T_{x} f^{\prime \prime \prime}= & f^{\prime \prime \prime}+\frac{1}{2} \sum_{k} d_{k}\left(y_{i}\right) b_{i}(x) b_{k} \\
& +\frac{1}{2} \sum_{i} d_{k}\left(y_{i}\right) b_{k}(x) b_{i}+\frac{1}{2} \sum d_{k}\left(y_{i}\right) b_{k}(x) b_{i}(x) 1 \\
= & f^{\prime \prime \prime}+\sum_{k} d_{k}(x) b_{k}+d^{\prime}(x) 1
\end{aligned}
$$

where $d^{\prime}=(1 / 2) \sum d_{k}\left(y_{i}\right) b_{k} b_{i}$. Thus

$$
T_{x}\left(f^{\prime \prime}-f^{\prime \prime \prime}\right)-\left(f^{\prime \prime}-f^{\prime \prime \prime}\right)=\left(d_{0}(x)-d^{\prime}(x) 1\right) .
$$

By Lemma $3 d_{0}-d^{\prime}$ is a continuous linear function. Then

$$
T_{x}\left(f^{\prime \prime}-f^{\prime \prime \prime}-\left(d_{0}-d^{\prime}\right)\right)-\left(f^{\prime \prime}-f^{\prime \prime \prime}-\left(d_{0}-d^{\prime}\right)\right)=0
$$

a.e., and thus by Lemma $2 f^{\prime \prime}-f^{\prime \prime \prime}-\left(d_{0}-d^{\prime}\right)$ is a constant almost everywhere. Since $f^{\prime \prime}=f+a$ polynomial, we conclude that $f$ is equal to a polynomial almost everywhere.

Lemma 6. Let $p$ be a measurable function on $G$ such that for all $x$ and $y, p(x+y)=p(x) p(y)$. Then $p$ is continuous.

Proof. Since $\ln |p(x)|$ is a measurable linear function, $p$ is locally bounded, by Lemma 1 . Let $E$ be an open set with compact closure. Then

$$
\int_{E} p(x-y) p(y) d y=p(x) \mu(E)
$$

so

$$
\mu(E)[p(x+\varepsilon)-p(x)]=\int_{E}[p(x+\varepsilon-y)-p(x-y)] p(y) d y,
$$

and

$$
\mu(E)|p(x+\varepsilon)-p(x)| \leqq \sup _{y \in E}|p(y)| \int_{E+x}|p(-t+\varepsilon)-p(-t)| d t .
$$

Since $p$ is locally bounded, the right side goes to zero as $\varepsilon$ goes to zero. Thus $p$ is continuous.

LEMMA 7. If $f$ is a measurable function on $G$ and if for all $x$ in $G, T_{x} f=p(x) f$ a.e., then $p$ is an exponential.

Proof. We first show that $p$ is measurable. If $n$ is a positive integer, define the function $g_{n}$ by

$$
g_{n}(x)= \begin{cases}f(x) & \text { if }|f(x)| \leqq n \\ 0 & \text { if }|f(x)|>n\end{cases}
$$


Let $h$ be an integrable function such that $f h$ is integrable and

Then

$$
\int f h \neq 0 \text {. }
$$

$$
\int\left(T_{x} f\right) h=p(x) \int f h .
$$

Define

$$
p_{n}(x)=\left[\int\left(T_{x} g_{n}\right) h\right] /\left(\int f h\right) .
$$

The function $p_{n}$ is continuous because $g_{n}$ is bounded and $h$ is integrable. For each $x, p_{n}(x)$ converges to $p(x)$ by the Lebesgue dominated convergence theorem. Since $p$ is the limit of continuous functions, $p$ is measurable. The function $p$ is multiplicative since $T_{x+y}=p(x+y)$ and also $T_{x+y} f=T_{x} T_{y} f=T_{x} p(y) f=p(y) T_{x} f=p(y) p(x) f$. By Lemma $6, p$ is continuous.

THEOREM 1. Let $V$ be a translation invariant finite dimensional complex linear space of measurable functions on a locally compact abelian group $G$. Then every function in $V$ is an exponential polynomial.

Proof. Since the family of translation operators is a commuting family of mappings from $V$ into itself, we can write $V$ as a direct sum $V^{1}+\cdots+V^{k}$ where each subspace $V^{j}$ is invariant and indecomposable under this family of mappings. Also a basis for $V$ can be found so that on $V^{j}$ the translation operators have matrices which are lower triangular with all diagonal elements equal, i.e., of the form

$$
\left[\begin{array}{ccc}
p_{j}(x) & & 0 \\
& \ddots & \\
* & & p_{j}(x)
\end{array}\right]
$$

(See Jacobson [3], p. 134). By Lemma 7, $p_{j}$ is an exponential function, since. there is a basis element $f_{j}$ such that $T_{x} f_{j}=p_{j}(x) f$. To simplify notation, we shall assume that $V$ itself is invariant and indecomposable so that we may omit the subscripts. Let $V^{\prime}=\left\{p^{-1} f: f \in V\right\}$. An easy computation shows that multiplication by an exponential takes translation invariant spaces into translation invariant spaces, so that $V^{\prime}$ is also invariant and indecomposable. Then in a suitable basis the matrix of translation has the form $*$. We can determine the diagonal elements by looking at a one dimensional subspace. Since the translates of $p^{-1} f$ span a one dimensional subspace if and only if the translates of $f$ do also, we have 


$$
\begin{aligned}
{\left[T_{y}\left(p^{-1} f\right)\right](x) } & =p^{-1}(x+y)\left(T_{y} f\right)(x) \\
& =p^{-1}(x) p^{-1}(y) p(y) f(x)=\left(p^{-1} f\right)(x) .
\end{aligned}
$$

This implies that in a suitable basis, the matrix of translation by any $x$ on $V^{\prime}$ is zero above the diagonal and identically equal to one on the diagonal. Applying Lemma 5 to the basis elements of $V^{\prime}$ in succession, first to the element $f_{1}$ such that the translates of $f_{1}$ span a one dimensional space, then to the element $f_{2}$ such that translates of $f_{2}$ and $f_{1}$ span a two dimensional space, and so on, we see that $V^{\prime}$ consists of polynomials, and that $V$ consists of polynomials multiplied by the exponential $p$.

Lemma 8. Suppose $p$ is an unbounded exponential function on $R^{n}$. Then (a) there is no nonzero function $f$ on $R^{n}$ with the properties: (1) $f$ has compact support, (2) $\hat{f}$ is integrable, (3) $p \hat{f}$ is integrable,

(b) there is no nonzero function $f$ with the properties (1) $f$ has compact support, (2) $f$ is square integrable, (3) p $\hat{f}$ is square integrable.

Proof. (a) Suppose there is a nonzero function $f$ with the three properties. Then we can assume $f(0) \neq 0$ without loss of generality. Since the hypotheses are satisfied for $p$ if and only if they are satisfied for $|p|$, we need only consider the case that $p$ is real. If so, $p(x)=$ $\exp (a, x)$ where $a$ is a real $n$-tuple. Define, for $t$ real,

$$
g(t)=f(t a)=\frac{1}{2 \pi} \int_{R^{n}} \exp i t(y, a) \hat{f}(y) d y .
$$

If $z$ is complex, $-1 \leqq \operatorname{Im}(z) \leqq 0$, define

$$
g(z)=\frac{1}{2 \pi} \int \exp i z(y, a) \hat{f}(y) d y .
$$

If $-1 \leqq \operatorname{Im}(z) \leqq 0$, then $|\exp i z(y, a) \hat{f}(y)| \leqq|\exp (y, a) \hat{f}(y)|+|\hat{f}(y)|$ so the integral defining $g(z)$ converges absolutely. If $-1<\operatorname{Im}(z)<0$, we can differentiate with respect to $z$ and see that $g(z)$ is analytic in this region. Thus $g(t)$ ( $t$ real) are the boundary values of an analytic function, so by the reflection principle, $g$ cannot vanish in an interval without vanishing identically. Since $g(0)=f(0) \neq 0$, and $g$ has compact support, we have a contradiction.

(b) Suppose there is a nonzero function $f$ with these properties. Then apply part (a) with $f$ replaced by $f * f$ and $p$ replaced by $p^{2}$. We conclude that $f * f=0$ which implies that $\hat{f}^{2}=0$ or $f=0$, which is a contradiction.

Lemma 9. Let $D$ be a closeable densely defined operator from 
$L^{2}\left(R^{n}\right)$ to itself that commutes with translations. Then there is a measurable function $b$ on $R^{n}$ so that for any $f$ in the domain of $D$, $D f=F^{-1} b F$ where $F$ denotes the fourier transform and $b$ denotes the operator of multiplication by the function $b$.

Proof. It is known that a closeable densely defined operator from $L^{2}\left(R^{n}\right)$ to itself that commutes with multiplication by bounded exponentials is multiplication by a measurable function. (See Kunze [4], p. 5). Apply this result to $F^{-1} D F$.

For the purpose of the following theorem, a differential difference operator with constant coefficients is an operator of the form $F^{-1} b F$ where $F$ denotes the fourier transform and $b$ denotes multiplication by an exponential polynomial $b$ with only bounded exponentials appearing.

THEOREM 2. Let $D$ be a closed densely defined operator from $L^{2}\left(R^{n}\right)$ into itself. Then $D$ is a differential difference operator with constant coefficients if and only if the following conditions are satisfied: (1) $D$ commutes with translations, (2) there is a nonzero function $f$ with compact support such that $f(x) e^{i(h, x)}$ is in the domain of $D$ for all $h$ in $R^{n}$, (3) the set of functions $\left\{e^{-i(h, x)} D e^{i(h, x)} f(x): h \in R^{n}\right\}$ spans a finite dimensional complex linear space of functions.

Proof. It is trivial that condition (1) is necessary. Condition (2) is necessary since we can take $f$ to be any infinitely differentiable function with compact support; and a direct calculation shows that condition (3) is necessary.

Lemma 9 and condition (1) imply that $D$ is of the form $F^{-1} b F$. Condition (3) implies that $\left\{\left(T_{x} b\right) \hat{f}: x \in R^{n}\right\}$ spans a finite dimensional space Since $\hat{f}$ is analytic, it is different from zero almost everywhere, so we can divide by it and conclude that $\left\{T_{x} b: x \in R^{n}\right\}$ spans a finite dimensional space $T(b)$ of measurable functions and thus, by Theorem $1, b$ is an exponential polynomial. Also, if $g$ is in $T(b)$, then $g \hat{f}$ is square integrable by condition (2). We have to show that $b$ contains only bounded exponentials. By the proof of Theorem 1, every exponential $p$ that appears in $b$ is also in the space $T(b)$, and thus $p \hat{f}$ is square integrable. But if $p$ is unbounded, then Lemma 8 gives a contradiction.

\section{REFERENCES}

1. P. M. Anselone and J. Korevaar, Translation invariant subspaces of finite dimension, Proc. Amer. Math. Soc. 15 (1964), 747-752.

2. L. Hörmander, Linear partial differential operators, Academic Press, New York, 1963.

3. N. Jacobson, Lectures in abstruct algebra, Vol. II, Van Nostrand, Princeton, N. J. 1957. 
4. R. A. Kunze, An operator theoretic approach to generalized fourier transforms, Ann. of Math. 69 (1959).

5. C. Loewner, On some transformation semigroups invariant under Euclidean or non-Euclidean isometries, J. Math. Mech. 8 (1959), 393-409.

6. J. J. Stone, Exponential polynomials on commutative semigroups, Appl. Math. and Stat. Lab. Technical Note No. 14, Stanford University, 1960.

Received December 20, 1968.

Mathematical Institute

AARHUS, DENMARK

AND

Wisconsin State UNIVERSITY-Whitewater 



\section{PACIFIC JOURNAL OF MATHEMATICS}

\section{EDITORS}

\author{
H. SAMELSON \\ Stanford University \\ Stanford, California 94305 \\ RICHARD PIERCE \\ University of Washington \\ Seattle, Washington 98105
}

J. DugundJI

Department of Mathematics University of Southern California Los Angeles, California 90007

BASIL GORDON*

University of California

Los Angeles, California 90024

\section{ASSOCIATE EDITORS}
E. F. BECKENBACH
B. H. NEUMANN
F. WOLE
K. YOSHIDA

\section{SUPPORTING INSTITUTIONS}

\author{
UNIVERSITY OF BRITISH COLUMBIA \\ CALIFORNIA INSTITUTE OF TECHNOLOGY \\ UNIVERSITY OF CALIFORNIA \\ MONTANA STATE UNIVERSITY \\ UNIVERSITY OF NEVADA \\ NEW MEXICO STATE UNIVERSITY \\ OREGON STATE UNIVERSITY \\ UNIVERSITY OF OREGON \\ OSAKA UNIVERSITY \\ UNIVERSITY OF SOUTHERN CALIFORNIA
}

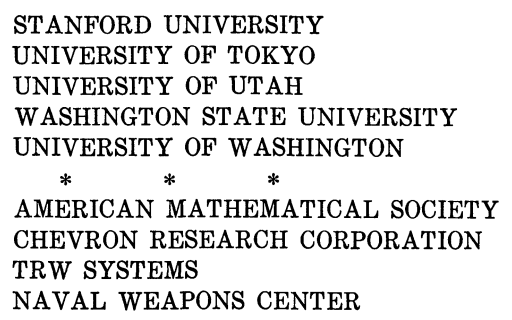

The Supporting Institutions listed above contribute to the cost of publication of this Journal, but they are not owners or publishers and have no responsibility for its content or policies.

Mathematical papers intended for publication in the Pacific Journal of Mathematics should be in typed form or offset-reproduced, (not dittoed), double spaced with large margins. Underline Greek letters in red, German in green, and script in blue. The first paragraph or two must be capable of being used separately as a synopsis of the entire paper. The editorial "we" must not be used in the synopsis, and items of the bibliography should not be cited there unless absolutely necessary, in which case they must be identified by author and Journal, rather than by item number. Manuscripts, in duplicate if possible, may be sent to any one of the four editors. Please classify according to the scheme of Math. Rev. 36, 1539-1546. All other communications to the editors should be addressed to the managing editor, Richard Arens, University of California, Los Angeles, California, 90024.

50 reprints are provided free for each article; additional copies may be obtained at cost in multiples of 50 .

The Pacific Journal of Mathematics is published monthly. Effective with Volume 16 the price per volume (3 numbers) is $\$ 8.00$; single issues, $\$ 3.00$. Special price for current issues to individual faculty members of supporting institutions and to individual members of the American Mathematical Society: $\$ 4.00$ per volume; single issues $\$ 1.50$. Back numbers are available.

Subscriptions, orders for back numbers, and changes of address should be sent to Pacific Journal of Mathematics, 103 Highland Boulevard, Berkeley, California, 94708.

PUBLISHED BY PACIFIC JOURNAL OF MATHEMATICS, A NON-PROFIT CORPORATION

Printed at Kokusai Bunken Insatsusha (International Academic Printing Co., Ltd.), 7-17, Fujimi 2-chome, Chiyoda-ku, Tokyo, Japan.

* Acting Managing Editor. 


\section{Pacific Journal of Mathematics}

\section{Vol. 32, No. $2 \quad$ February, 1970}

Harry P. Allen and Joseph Cooley Ferrar, Jordan algebras and exceptional subalgebras of the exceptional algebra $E_{6} \ldots \ldots \ldots \ldots \ldots \ldots \ldots 283$

David Wilmot Barnette and Branko Grünbaum, Preassigning the shape of a

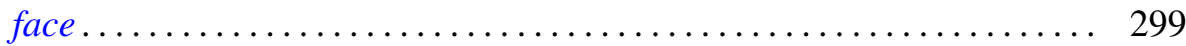

Robert Francis Craggs, Involutions of the 3-sphere which fix 2-spheres . . . . 307

David William Dean, Bor-Luh Lin and Ivan Singer, On k-shrinking and $k$-boundedly complete bases in Banach spaces ................ 323

Martin Engert, Finite dimensional translation invariant subspaces ....... 333

Kenneth Lewis Fields, On the global dimension of residue rings ......... 345

Howard Gorman, The Brandt condition and invertibility of modules ....... 351

Benjamin Rigler Halpern, A characterization of the circle and interval ..... 373

Albert Emerson Hurd, A uniqueness theorem for second order quasilinear

hyperbolic equations ............................... 415

James Frederick Hurley, Composition series in Chevalley algebras ...... 429

Meira Lavie, Disconjugacy of linear differential equations in the complex

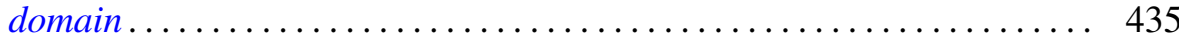

Jimmie Don Lawson, Lattices with no interval homomorphisms ......... 459

Roger McCann, A classification of center-foci ................. 467

Evelyn Rupard McMillan, On continuity conditions for functions . . . . . . . 479

Graciano de Oliveira, A conjecture and some problems on permanents .... 495

David L. Parrott and S. K. Wong, On the Higman-Sims simple group of order $44,352,000$.

Jerome L. Paul, Extending homeomorphisms ................. 517

Thomas Benny Rushing, Unknotting unions of cells .............. 521

Peter Russell, Forms of the affine line and its additive group.......... 527

Niel Shilkret, Non-Archimedean Gelfand theory ................. 541

Alfred Esperanza Tong, Diagonal submatrices of matrix maps.......... 551 